\title{
A monolithic model for phase-field fracture and waves in solid-fluid media towards earthquakes ${ }^{1}$
}

\author{
TOMÁŠ ROUBÍČEK ${ }^{3}$ AND ROMAN VODIČKA ${ }^{4}$.
}

\begin{abstract}
Coupling of rupture processes in solids with waves also propagating in fluids is a prominent phenomenon arising during tectonic earthquakes. It is executed here in a single 'monolithic' model which can asymptotically capture both damageable solids (rocks) and (visco-)elastic fluids (outer core or oceans). Both ruptures on pre-existing lithospheric faults and a birth of new faults in compact rocks are covered by this model, together with emission and propagation of seismic waves, including, e.g., reflection of S-waves and refraction of P-waves on the solid-fluid interfaces. A robust, energy conserving, and convergent staggered FEM discretisation is devised. Using a rather simplified variant of such models for rupture, three computational experiments documenting the applicability of this approach are presented. Some extensions of the model towards more realistic geophysical modelling are outlined, too.
\end{abstract}

Keywords: Fracture of faults, tectonic earthquake dynamics, elastic waves, elastic-fluid/solid interaction, numerical modelling

PACS: 46.50.+a, 91.30.Cd, 91.30.Px

AMS Class.: 74F10, 74J10, 74R20, 74S05, 86-08

\section{Introduction}

Dynamic fracture mechanics is an area of continuum mechanics of solids with wide applications in engineering and particularly also in geophysics. Global geophysical models typically deal with several very different phenomena and couple various models due to the layered character of terrestrial planets (including our planet Earth as well as our Moon). This paper demonstrates the philosophy that a single model can be used instead of several specialized models that otherwise would need to be mutually coupled in a rather complicated way. Such a single universal (we say "monolithic") model can also be straightforwardly implemented on computers omitting any interfaces which usually complicate implementations. In particular, the transient conditions on fluid/solid interfaces are automatically involved and need not be specified. This can be a sound advantage of the presented model. Of course, computationally, such a monolithic-type model may not always make it easier to produce really relevant simulations on the computers we have at our disposal nowadays. Routine calculations are performed separately either for earthquake sources locally (see, e.g., [10]) or for purely seismic global 3D models, cf., e.g., [25, 26, 29,46]. Their mutual coupling is already treatable well in the literature $[18,20,21,36]$. Therefore, there is promising potential that the presented coupling monolithic approach may become even more amenable in the future with ever increasing computer efficiency.

The phenomena we have in mind in this paper involve global seismicity and tectonics. In particular the latter involves, e.g., ruptures of lithospheric faults or a birth of new faults, generating seismic waves which then propagate through the solid-like silicate mantle and iron-nickel inner core both in the shear (S) or the pressure (P) modes. In contrast to the P-waves (also called primary or compressional waves), the S-waves (also called secondary waves) are suppressed in the fluidic iron-nickel outer core and also in the water oceans.

A very low attenuation of seismic waves is the ultimate phenomenon. We, therefore, take the Jeffrey's rheology model (i.e., a serial combination of the Maxwell and Kelvin-Voigt rheology as in Figure 1/bottom-left, cf., e.g., [33] or also [39]) as a basic global "monolithic" ansatz. In various limits in the deviatoric and the volumetric parts, we model different parts of planet Earth. Jeffrey's rheology also seems more realistic in particular because it covers (in the limit) also the Kelvin-Voigt model applied to the volumetric strain (actually considered as a starting model in Figure 1/left) whereas the pure Maxwell rheology, allowing for big creep during long geological periods, is not a relevant effect in the volumetric part.

\footnotetext{
${ }^{1}$ The support from the grants 17-04301S (as far as dissipative evolutionary aspects concerns) and 19-04956S (as far as modelling of dynamic and nonlinear behaviour concerns) of the Czech Sci. Foundation and VEGA 1/0078/16 of the Ministry of Education, Science, Research and Sport of the Slovak Republic, and the institutional support RVO:61388998 (С̆R) are acknowledged.

${ }^{2}$ Institute of Thermomechanics, Czech Academy of Sciences, Dolejškova 5, CZ-182 00 Praha 8, Czech Republic.

${ }^{3}$ Mathematical Institute, Charles University, Sokolovská 83, CZ-186 75 Praha 8, Czech Republic

${ }^{4}$ Civil Engineering Faculty, Technical University of Košice, Vysokoškolská 4, SK-042 00 Košice, Slovakia
} 
Respecting the solid parts of the model, we use the Lagrangian description even in the fluid regions, i.e. here all equations are formulated in terms of displacements rather than velocities. The reference and the actual space configurations automatically coincide with each other in our small strain (and small displacement) ansatz, which is well relevant in geophysical short-time scales of seismic events.

In the solid-like part, various inelastic processes are considered to model tectonic earthquakes on lithospheric faults together with long-lasting healing periods in between them, as well as aseismic slips, and various other phenomena. To this goal, many internal variables may be involved such as aging/damage, inelastic strain, porosity, water content, breakage (i.e., essentially like another damage-like internal variable in modeling of granular materials), and temperature, cf. [30,31,33]. On the other hand, those sophisticated models are focused rather on local events around the tectonic faults without ambitions to be directly coupled with the global seismicity. Here, rather for the lucidity of the exposition, we reduce the set of internal variables to only one scalar variable and one matrix-valued variable, namely damage/aging and an inelastic strain, respectively. The calculations in Sect. 5 involve the only former one. Even this simple scenario, however, has a capacity to trigger a spontaneous rupture (so-called dynamic triggering) with emission of seismic waves and, in a certain simplification, can serve as a seismic source coupled with the overall global model. The mentioned inelastic strain can capture Maxwell-type rheologies relevant in the solid mantle and inner core to capture long-term creep (aseismic) effects up to $10^{5}$ yrs.

Let us emphasize that the usual models are focused only on either the propagation of seismic waves along the whole globe while their source is considered given, or on the description of seismic sources due to tectonic events, but not their mutual coupling. If a coupling is considered, then it concerns rather local models not considering the layered structure of the whole planet, cf. e.g. [5,6,20,21,32]. The reality ultimately captures very different mechanical properties of different layers of the Earth, in particular the mantle and the inner core which are solid on the short-time scales versus the outer core and the oceans which are fluidic even on the short-time scales. Some other coupled models implement a pre-existing fault (in contrast to our model allowing for new faults birth, cf. Section 5.2) and use a slip-dependent friction law [45].

The goal of this contribution is threefold:

$\alpha)$ to present a model that might simultaneously capture the sources of seismic waves that necessarily behave nonlinearly (like ruptures of tectonic faults) and the propagation of seismic waves possibly even over the whole planet (when further special algorighmic and computational methods would be used, not handled in this article, however), both phenomena being mutually coupled.

$\beta$ ) by proper scaling to approximate viscoelastic (so-called Boger's [7]) or merely elastic fluids that are relevant in the outer core and in the oceans (with a very low or just zero viscosity) where S-waves cannot propagate while Pwaves are only refracted on the solid/fluid interfaces (in particular on Guttenberg's core-mantle discontinuity).

$\gamma)$ to document computational efficiency of the monolithic model at least on 2-dimensional rather local simulations of various, quite distinct geophysical events.

We refer to [40] for the rigorous analysis as far as asymptotics of the scaling of viscosities in even a more complex model involving also self-induced gravity, tidal, Coriolis, and centrifugal forces; cf. Sect. 6.2.

\section{Energy-based modelling approach}

Mechanical models in general (and those used in geophysics in particular) typically are (or should be) believed to be governed by energies and, most often, in a way that the conservative and the dissipative parts are separated. In the isothermal variant, the systems have a simple general structure of an abstract dissipative dynamic equation

$$
\mathscr{M}^{\prime} \ddot{q}+\mathscr{R}_{\dot{q}}^{\prime}(q, \dot{q})+\mathscr{E}^{\prime}(q)=\mathscr{F}(t)
$$

with a kinetic energy $\mathscr{M}$, a (pseudo) potential of dissipative forces $\mathscr{R}(q, \cdot)$, a stored energy $\mathscr{E}$, and external forcing $\mathscr{F}$ as a time-dependent functions of the state $q$ in the reference domain $\Omega$. In (1), $\mathscr{R}_{\dot{q}}^{\prime}$ denotes the partial differential of $\mathscr{R}=\mathscr{R}(q, \dot{q})$ with respect to $\dot{q}$. In fact, $\mathscr{R}(q, \cdot)$ may be non-differentiable at $\dot{q}=0$ when some activated processes (like damage or plasticity) occur so that $\mathscr{R}_{\dot{q}}^{\prime}(q, \cdot)$ may be set-valued and (1) is to be an inclusion rather than equality; yet we will ignore these technicalities in this presentation. 
This state $q$ typically involves, beside of the displacement, also some internal variables like the inelastic strains describing creep and a "permanent" deformation resulting from re-occurring shifts during earthquakes, damage/aging, etc., complying with the concept of generalized standard materials with internal variables [15]. In (1), we use the notation $\dot{q}=\frac{\mathrm{d}}{\mathrm{d} t} q$ and $\ddot{q}=\frac{\mathrm{d}^{2}}{\mathrm{~d} t^{2}} q$, and $(\cdot)^{\prime}$ denotes the differential. Also, (1) takes the structure of dissipative Hamiltonian system, and the Hamilton variational principle [17] extended to dissipative systems as in [3] says that the solution $q$ to (1) on a fixed time interval $[0, T]$ is a critical point of the integral functional

$$
q \mapsto \int_{0}^{T} \mathscr{M}(\dot{q})-\mathscr{E}(q)-\langle\mathfrak{f}, q\rangle \mathrm{d} t
$$

with a nonconservative force $\mathfrak{f}=\mathscr{R}_{\dot{q}}^{\prime}(q, \dot{q})-\mathscr{F}(t)$ considered fixed on the affine manifold respecting some initial or terminal conditions. The notation $\langle\cdot, \cdot\rangle$ in (2) means the value of a functional (in our case $\mathfrak{f}$ ) on a test function (in our case $q$ ), which for sufficiently smooth functions can be understood as an integral, see e.g. (9d) below.

This energy-governed structure (1) allows to control the energetics: indeed, testing (1) by $\dot{q}$ and using the calculus $\left\langle\mathscr{M}^{\prime} \ddot{q}, \dot{q}\right\rangle=\frac{\mathrm{d}}{\mathrm{d} t} \mathscr{M}(\dot{q})$ and $\left\langle\mathscr{E}^{\prime}(q), \dot{q}\right\rangle=\frac{\mathrm{d}}{\mathrm{d} t} \mathscr{E}(q)$, we arrive (at least formally) to the energy balance on a time interval $[0, t]:$

$$
\underbrace{\mathscr{M}(\dot{q}(t))+\mathscr{E}(q(t))}_{\begin{array}{c}
\text { kinetic }+ \text { stored energy } \\
\text { at time } t
\end{array}}+\underbrace{\int_{0}^{t} \Xi(q(s), \dot{q}(s)) \mathrm{d} s}_{\begin{array}{c}
\text { dissipated energy over } \\
\text { the time interval }[0, t]
\end{array}}=\underbrace{\mathscr{M}(\dot{q}(0))+\mathscr{E}(q(0))}_{\begin{array}{c}
\text { kinetic+stored energy } \\
\text { at time } 0
\end{array}}+\underbrace{\int_{0}^{t}\langle\mathscr{F}(s), \dot{q}(s)\rangle \mathrm{d} s}_{\begin{array}{c}
\text { work done by loading } \\
\text { over time interval }[0, t]
\end{array}}
$$

with the dissipation rate

$$
\Xi(q, \dot{q})=\left\langle\mathscr{R}_{\dot{q}}^{\prime}(q, \dot{q}), \dot{q}\right\rangle .
$$

Moreover, this structure allows for various numerically stable time discretisations, and for rigorous analysis as far as convergence and existence of solutions to (1) concerns.

\section{Coupling of rupturing solids with elastic fluids}

Denoting the displacement by $u$, we use the usual concept of small strains, with the total strain $e=e(u)=\frac{1}{2} \nabla u+$ $\frac{1}{2}(\nabla u)^{\top}$ and the inelastic, symmetric, trace-free strain $\pi$. As standard in small-strain theories, we consider the additive split, sometimes called Green-Naghdi split [13], of the total strain $e=e(u)$ as

$$
e(u)=e_{\mathrm{el}}+\pi
$$

with $e_{\mathrm{el}}$ denoting the elastic strain and $\pi$ the inelastic strain, the latter being considered as trace free. To distinguish the response on the shear or compression load, we further introduce its orthogonal decomposition to the spherical and the deviatoric part:

$$
\begin{aligned}
& e(u)=\operatorname{sph} e(u)+\operatorname{dev} e(u) \quad \text { with } \operatorname{dev} e=e-\frac{\operatorname{tr} e}{3} \mathbb{I}, \\
& \operatorname{dev} e=\operatorname{dev}\left(e_{\mathrm{el}}+\pi\right)=e_{\operatorname{dev}}+\pi \text { with } e_{\operatorname{dev}}=\operatorname{dev} e_{\mathrm{el}},
\end{aligned}
$$

where $\mathbb{I}$ is the identity matrix while tre $e=\sum_{i=1}^{3} e_{i i}$ denotes the trace of $e$.

\subsection{Solid model for mantle and inner core}

A rather minimal scenario for a simplified seismic source model in the solid part (the mantle) is an isothermal damage (also called aging) in the deviatoric part. To model also the aseismic slip (creep) or a permanent displacement during cumulation of subsequent shift (which is important in particular if healing is considered in between re-occurring earthquakes), as well as a low attenuation of seismic waves, the Maxwell viscoelastic rheology is considered in the deviatoric response. For well-posedness of the problem, it is suitable to combine it with the Kelvin-Voigt rheology, which results to the Jeffreys rheological model, as used in [33]. The spherical response in the solid part (the mantle 


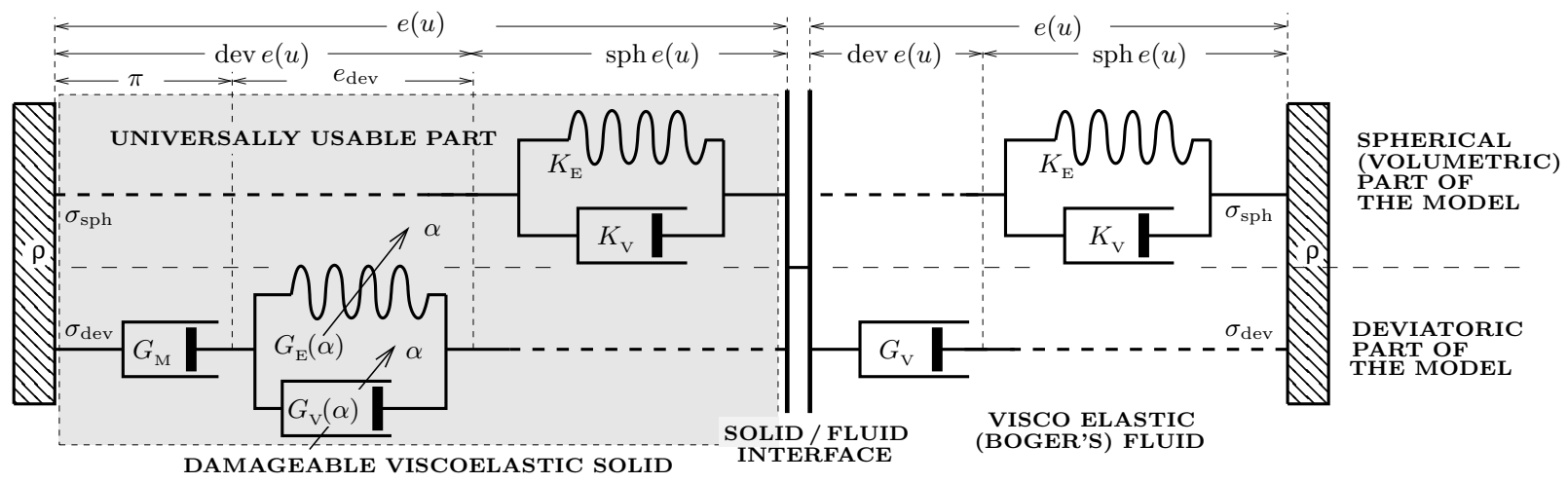

Figure 1: Schematic diagram for solid-fluid interaction. The spherical response is the Kelvin-Voigt rheology both in the solid and in the fluid parts. The deviatoric response of the solid uses a viscoelastic Jeffreys rheology subjected to damage $\alpha$, while the fluid uses the mere Stokes model.

and inner core) exhibits just the undamageable Kelvin-Voigt rheology, reflecting the phenomenon that neither any big permanent deformation nor damage by compression is possible. Cf. Figure 1/left for the overall rheological model.

The state $q=(u, \pi, \alpha)$ then involves the displacement $u$, the (trace-free) inelastic strain $\pi$, and the scalar damage variable $\alpha$; alternatively, $\alpha$ is called also aging and we follow the usual convention that $\alpha=0$ means no damage while $\alpha=1$ is the maximal degradation. Consistently with Section 2, we must specify the energies governing the model through the (generalized) Hamiltonian principle (2). The specific stored energy is postulated as

$$
\varphi(e, \pi, \alpha)=\frac{3}{2} K_{\mathrm{E}}|\operatorname{sph} e|^{2}+G_{\mathrm{E}}(\alpha)\left|e_{\mathrm{dev}}\right|^{2}+\gamma_{\mathrm{DAM}}(\alpha)=\frac{3}{2} K_{\mathrm{E}}|\operatorname{sph} e|^{2}+G_{\mathrm{E}}(\alpha)|\operatorname{dev} e-\pi|^{2}+\gamma_{\mathrm{DAM}}(\alpha)
$$

with $K_{\mathrm{E}}$ the elastic bulk modulus and $G_{\mathrm{E}}=G_{\mathrm{E}}(\alpha)$ the elastic shear modulus introduced for a damageable Lamé material, and $\gamma_{\text {DAM }}=\gamma_{\text {DAM }}(\alpha)$ damage stored energy (also understood as the damage toughness). The specific dissipation potential is

$$
\begin{aligned}
& \zeta(\alpha ; \dot{e}, \dot{\pi}, \dot{\alpha})=\zeta_{\mathrm{VSC}}(\alpha ; \dot{e}, \dot{\pi})+\zeta_{\mathrm{DAM}}(\dot{\alpha}) \text { with } \\
& \zeta_{\mathrm{VSC}}(\alpha ; \dot{e}, \dot{\pi})=\frac{3}{2} K_{\mathrm{V}}|\operatorname{sph} \dot{e}|^{2}+G_{\mathrm{V}}(\alpha)|\operatorname{dev} \dot{e}-\dot{\pi}|^{2}+G_{\mathrm{M}}|\dot{\pi}|^{2}
\end{aligned}
$$

and with $\zeta_{\mathrm{DAM}}=\zeta_{\mathrm{DAM}}(\dot{\alpha}) \geq 0$ a convex function of the damage rate and with the convex quadratic functional $\zeta_{\mathrm{VSC}}$. The former one is the potential of non-conservative forces related to damage evolution while the latter one together with elastic parts in the stored energy introduces Kelvin-Voigt rheology for the spherical part and Jeffrey rheology for the deviatoric part. We consider still the specific kinetic energy in the usual form $\frac{\rho}{2}|\dot{u}|^{2}$ with $\rho=\rho(x)$ the mass density.

To introduce a length-scale into the model that allows for "controlling" a typical width of the damaged zone around fault core (usually varying in between 1-100 m) and of the narrow cataclasite fault core (i.e. the principal slip zone, usually varying in between $0.1-10 \mathrm{~m}$ ) where the inelastic strain is accommodated, the gradient theories are used. This means that $\varphi$ from (6) is augmented both by $|\nabla \alpha|^{2}$ and $|\nabla \pi|^{2}$ terms. In a special choice $\gamma_{\text {DAM }}(\alpha)=G_{\mathrm{c}} \frac{1}{2 \varepsilon} \alpha^{2}$ together with $G_{\mathrm{E}}(\alpha)=\left(\varepsilon^{2} / \varepsilon_{0}^{2}+(1-\alpha)^{2}\right) G_{0}$ where $G_{0}$ is the shear modulus of the undamaged material (up to a small value of $\varepsilon^{2} / \varepsilon_{0}^{2}$ ) and $\varepsilon>0$ small (with respect to $\varepsilon_{0}$ ), the "augmented" specific stored energy $\varphi_{\mathrm{A}}$ takes the form

$$
\varphi_{\mathrm{A}}(e, \pi, \alpha, \nabla \pi, \nabla \alpha):=\frac{3}{2} K_{\mathrm{E}}|\operatorname{sph} e(u)|^{2}+\left(\frac{\varepsilon^{2}}{\varepsilon_{0}^{2}}+(1-\alpha)^{2}\right) G_{0}|\operatorname{dev} e(u)-\pi|^{2}+\frac{\kappa}{2}|\nabla \pi|^{2}+\underbrace{G_{\mathrm{c}}\left(\frac{1}{2 \varepsilon} \alpha^{2}+\frac{\varepsilon}{2}|\nabla \alpha|^{2}\right)}_{\text {crack surface density }}
$$

with $\varepsilon_{0}>0$ (dimension $[\mathrm{m}]$ ), $\kappa$ (dimension $[\mathrm{J} / \mathrm{m}]$ ) allows to control the width of the mentioned cataclasite fault core while $\varepsilon$ (dimension $[m]$ ) allows to control the width of the mentioned damaged zone around the fault core, and a so-called fracture toughness $G_{\mathrm{c}}>0$ (dimension $\left[\mathrm{J} / \mathrm{m}^{2}\right]$ ) fixed. This is, in fact, the usual phase-field crack model, 
here considered only in the deviatoric component reflecting the phenomenon that pure tension (and Mode I cracks) is avoided in geophysically relevant situations while compression does not lead directly to cracks, so that only shear may lead to rupture (in Mode II). In the static situation, for $\varepsilon$ converging to zero, this so-called Ambrosio-Tortorelli functional approximates the Griffith crack model [14], originally shown in the scalar case [1] and later for the vectorial case by [12]. In the dynamical case, this approximation property is not rigorously justified, but nevertheless this phase-field model is routinely used, cf. e.g. $[8,28,35,41]$. Such a crack model is relevant particularly if a new fault is nucleated in the compact rock, although it is a rare event geophysically.

\subsection{Fluid model for outer core and oceans}

Besides solid regions, there are layers in our planet that are naturally fluidic, namely the outer core and, of course, the oceans. From the seismic wave propagation, they must exhibit some spherical elastic response (otherwise, ideally, incompressible fluids would lead to unphysically infinitely large P-wave speed) but no deviatoric elastic response to prevent S-wave propagation in such regions. In any case, damage becomes irrelevant in these regions.

One scenario is to keep still a (presumably small) viscous response both in the spherical and the deviatoric part, leading respectively to a combination of the Kelvin-Voigt solid rheology and the Stokes fluid rheology. In the notation from Section 3.1, this means that we take $G_{\mathrm{E}}=0, \gamma_{\mathrm{DAM}}=0, G_{\mathrm{V}}>0$ independent of $\alpha, \kappa=0, G_{\mathrm{c}}=0$, and we formally put $G_{\mathrm{M}}=\infty$. The last action results to $\pi=0$ provided the initial conditions are $\left.\pi\right|_{t=0}=0$. In fact, an equivalent option would be to put $G_{\mathrm{V}}=\infty$ and keep $G_{\mathrm{M}}>0$ finite (small). This leads to the so-called viscoelastic (Boger's [7]) fluid, cf. Figure 1/right-part. The S-waves actually can slightly penetrate into such fluids but are soon attenuated and thus cannot propagate practically.

Another scenario is to suppress any viscosity, obtaining, thus, the merely elastic fluid. This can be achieved, in addition to taking $G_{\mathrm{E}}=0, \gamma_{\mathrm{DAM}}=0, \kappa=0$, and $G_{\mathrm{c}}=0$, by putting both $K_{\mathrm{V}}=0$ and $G_{\mathrm{V}}=0$. Such fluids are fully conservative, leading to a linear hyperbolic problem. The S-waves cannot propagate through such fluidic regions at all.

\subsection{The coupled equations}

Let us consider the reference domain $\Omega$ occupied by a viscoelastic body in question (e.g., the whole planet Earth). Of course, the local potentials $\varphi_{\mathrm{A}}$ and $\zeta$, and also the mass density $\rho$ are allowed (and supposed) to depend on $x \in \Omega$, and in particular, they can vary on the solid and the fluid domains; let us denote them by $\Omega_{\mathrm{S}}$ and $\Omega_{\mathrm{F}}$, respectively. We will not indicate this dependence explicitly for notational simplicity.

Taking into account the additive split (4), the overall functionals used in Section 2 are now:

$$
\begin{aligned}
& \mathscr{E}(q)=\mathscr{E}(u, \pi, \alpha)=\int_{\Omega} \varphi_{\mathrm{A}}(e(u), \pi, \alpha, \nabla \pi, \nabla \alpha) \mathrm{d} x, \\
& \mathscr{R}(q, \dot{q})=\mathscr{R}(\alpha ; \dot{u}, \dot{\pi}, \dot{\alpha})=\int_{\Omega} \zeta_{\mathrm{VSC}}(\alpha ; e(\dot{u}), \dot{\pi})+\zeta_{\mathrm{DAM}}(\dot{\alpha}) \mathrm{d} x, \\
& \mathscr{M}(\dot{q})=\mathscr{M}(\dot{u})=\int_{\Omega} \frac{\rho}{2}|\dot{u}|^{2} \mathrm{~d} x, \\
& \langle\mathscr{F}(t), u\rangle=\int_{\Omega} g(t) \cdot u \mathrm{~d} x
\end{aligned}
$$

where $g$ is a bulk force, typically the gravitational force independent of time or some more general force as, e.g., in (23) or (24) below. Note the (9d) does not involve the internal variables $(\pi, \alpha)$, in accord with the conventional concept that the internal variables are not directly subjected to outer forcing.

More specifically, the system that results from (1) by considering (9) together with (8) looks as:

$$
\begin{aligned}
\rho \ddot{u}-\operatorname{div}\left(\sigma_{\mathrm{sph}}+\sigma_{\mathrm{dev}}\right)=g & \text { in } \Omega \\
\text { with } \sigma_{\mathrm{sph}}=3 K_{\mathrm{v}} \operatorname{sph} e(\dot{u})+3 K_{\mathrm{E}} \operatorname{sph} e(u) & \text { in } \Omega, \\
\text { and } \sigma_{\mathrm{dev}}=\left\{\begin{array}{cc}
2 G_{\mathrm{v}}(\alpha)(\operatorname{dev} e(\dot{u})-\dot{\pi}) & \\
+2 G_{\mathrm{E}}(\alpha)(\operatorname{dev} e(u)-\pi) & \text { in } \Omega_{\mathrm{S}}, \\
2 G_{\mathrm{v}} \operatorname{dev} e(\dot{u}) & \text { in } \Omega_{\mathrm{F}},
\end{array}\right. & \\
G_{\mathrm{M}} \dot{\pi}=\sigma_{\mathrm{dev}}+\operatorname{div}(\kappa \nabla \pi) & \text { in } \Omega_{\mathrm{S}},
\end{aligned}
$$




$$
\zeta_{\mathrm{DAM}}^{\prime}(\dot{\alpha})+G_{\mathrm{E}}^{\prime}(\alpha)|(\operatorname{dev} e(u)-\pi)|^{2}+\frac{G_{\mathrm{c}}}{\varepsilon} \alpha=\operatorname{div}\left(\varepsilon G_{\mathrm{c}} \nabla \alpha\right) \quad \text { in } \Omega_{\mathrm{S}}
$$

Note that the spherical and the deviatoric stresses are orthogonal, i.e. $\sigma_{\mathrm{sph}}: \sigma_{\mathrm{dev}}=0$. Also note that $\pi$ and $\alpha$ are relevant only on the solid domain $\Omega_{\mathrm{s}}$, cf. also Figure 1 .

There are no transient conditions for displacement/stress on the solid-fluid interior interfaces (i.e. between outer core and mantle and inner core and possibly also between mantle and oceans) because (10a) is considered on the whole domain $\Omega$. In particular, the stress-vector equilibrium and continuity of the displacement across these interfaces are automatically involved and does not need to be written explicitly. On the other hand, there are boundary conditions to be read from the abstract equation (1), namely

$$
\begin{array}{ll}
\left(\sigma_{\mathrm{sph}}+\sigma_{\mathrm{dev}}\right) \vec{n}=0 & \text { on } \partial \Omega, \\
\kappa \frac{\partial \pi}{\partial \vec{n}}=0 \quad \text { and } \quad \varepsilon G_{\mathrm{c}} \frac{\partial \alpha}{\partial \vec{n}}=0 & \text { on } \partial \Omega_{\mathrm{s}},
\end{array}
$$

where $\vec{n}$ denotes the normal to the boundary $\partial \Omega$ of $\Omega$ (e.g. the surface of the Earth) the interior boundaries $\partial \Omega_{\mathrm{S}}$ (i.e. on the mantle/core and mantle/oceans and inner/outer-core interfaces) and $\frac{\partial}{\partial \vec{n}}=\vec{n} \cdot \nabla$ denotes normal derivative.

We have in mind an initial-value problem, so that we have to complete the system still by initial conditions:

$$
\begin{array}{lll}
\left.u\right|_{t=0}=u_{0} & \text { and }\left.\quad \dot{u}\right|_{t=0}=v_{0} & \text { in } \Omega, \\
\left.\pi\right|_{t=0}=\pi_{0} & \text { and }\left.\quad \alpha\right|_{t=0}=\alpha_{0} & \text { in } \Omega_{\mathrm{S}} .
\end{array}
$$

Although it is usually not an aspect under attention in geophysical modelling, let us mention that it is possible to prove rigorously that, under a suitable data qualification, the initial-boundary-value problem (10)-(12) has a solution which also satisfies the energy conservation (3), cf. [40] for the case $G_{\mathrm{v}}$ independent of $\alpha$ while [27, Sect. 7.5] outlines modifications if $G_{\mathrm{v}}(\cdot)=\tau_{\mathrm{v}} G_{\mathrm{E}}(\cdot)$ for some relaxation time $\tau_{\mathrm{v}}=\tau_{\mathrm{v}}(x)>0$.

\subsection{Towards fluids from solids: a monolithic model}

One can approximate the fluid models from Sect. 3.2 asymptotically when sending corresponding parameters of the solid model in Sect. 3.1 to their limits. More specifically, Boger's viscoelastic fluid can be approached from the Jeffreys solid by sending

$$
G_{\mathrm{M}} \rightarrow \infty, G_{\mathrm{E}} \rightarrow 0, \kappa \rightarrow 0, \text { and } \zeta_{\mathrm{DAM}} \rightarrow 0 \text { in } \Omega_{\mathrm{F}} .
$$

When $\pi_{0}=0$ in (12b), the inelastic strain $\pi$ converges to 0 on the fluidic domain $\Omega_{\mathrm{F}}$. Under suitable data qualification and scaling, this convergence can rigorously be justified together with convergence of the energy balance, cf. [40].

Sending further

$$
G_{\mathrm{V}} \rightarrow 0 \text { and } K_{\mathrm{V}} \rightarrow 0 \text { in } \Omega_{\mathrm{F}},
$$

we approach the merely elastic fluid in $\Omega_{\mathrm{F}}$. Again, this convergence can rigorously be proved together with convergence of the energy balance (i.e. the energy dissipated via viscous attenuation in the Bogger fluid actually converges to zero) but for a slightly modified model with the bounded elastic stress and constant $G_{\mathrm{V}}$ in the solid part $\Omega_{\mathrm{S}}$, cf. [40].

The idea behind the "monolithic" approach is to take, instead of the limit fluid in Sect. 3.2, its approximation and to implement computationally only the solid model in the whole domain $\Omega$. Again, the interface conditions between $\Omega_{\mathrm{S}}$ and $\Omega_{\mathrm{F}}$ expressing here continuity of displacements and the traction stresses will thus be covered automatically without any extra effort in coding.

\section{Staggered / FEM discretisation}

We write the abstract equation (1) as a first-order system, which is more suitable for time discretisation than the original 2nd-order system, in particular because it allows for varying time steps during simulations. In view of (9), it has the structure

$$
\dot{u}=v,
$$




$$
\begin{aligned}
& \mathscr{M}^{\prime} \dot{v}+\mathscr{R}_{v}^{\prime}(\alpha ; v, \dot{\pi})+\mathscr{E}_{u}^{\prime}(u, \pi, \alpha)=\mathscr{F}(t), \\
& \mathscr{R}_{\dot{\pi}}^{\prime}(\alpha ; v, \dot{\pi})+\mathscr{E}_{\pi}^{\prime}(u, \pi, \alpha)=0, \\
& \mathscr{R}_{\dot{\alpha}}^{\prime}(\dot{\alpha})+\mathscr{E}_{\alpha}^{\prime}(u, \pi, \alpha)=0 .
\end{aligned}
$$

It is important that $\mathscr{R}$ is additively split, cf. (9b), which suggests the splitting with the time discretisation of the state $q$ to the components $(u, \pi)$ and $\alpha$ and in the same manner also the calculation is performed. Although $\mathscr{E}$ is necessarily nonconvex to facilitate modelling of sudden rupture events, it is also advantageous that both $\mathscr{E}(\cdot, \cdot, \alpha)$ and $\mathscr{E}(u, \pi, \cdot)$ are convex or, here in the ansatz (8), even quadratic.

Considering a time step $\tau=\left(\tau_{k}\right)_{k \in \mathbb{N}}$ with $k=1,2, \ldots$ indexing time levels $t^{k}$ in the time-discrete system so that $\tau^{k}=t^{k}-t^{k-1}$, and using also the Crank-Nicholson mid-point strategy, we devise the staggered (also called fractionalstep splitting) system

$$
\begin{aligned}
& \frac{u_{\tau}^{k}-u_{\tau}^{k-1}}{\tau_{k}}=v_{\tau}^{k-1 / 2} \text { with } v_{\tau}^{k-1 / 2}=\frac{v_{\tau}^{k}+v_{\tau}^{k-1}}{2}, \\
& \mathscr{M}^{\prime} \frac{v_{\tau}^{k}-v_{\tau}^{k-1}}{\tau_{k}}+\mathscr{R}_{v}^{\prime}\left(\alpha_{\tau}^{k-1} ; v_{\tau}^{k-1 / 2}, \frac{\pi_{\tau}^{k}-\pi_{\tau}^{k-1}}{\tau_{k}}\right)+\mathscr{E}_{u}^{\prime}\left(u_{\tau}^{k-1 / 2}, \pi_{\tau}^{k-1 / 2}, \alpha_{\tau}^{k-1}\right)=\mathscr{F}\left(t^{k}\right), \\
& \mathscr{R}_{\dot{\pi}}^{\prime}\left(\alpha_{\tau}^{k-1} ; v_{\tau}^{k-1 / 2}, \frac{\pi_{\tau}^{k}-\pi_{\tau}^{k-1}}{\tau_{k}}\right)+\mathscr{E}_{\pi}^{\prime}\left(u_{\tau}^{k-1 / 2}, \pi_{\tau}^{k-1 / 2}, \alpha_{\tau}^{k-1}\right)=0, \\
& \mathscr{R}_{\dot{\alpha}}^{\prime}\left(\frac{\alpha_{\tau}^{k}-\alpha_{\tau}^{k-1}}{\tau_{k}}\right)+\mathscr{E}_{\alpha}^{\prime}\left(u_{\tau}^{k}, \pi_{\tau}^{k}, \alpha_{\tau}^{k-1 / 2}\right)=0 .
\end{aligned}
$$

The system (16) is to be solved, recursively, for $k=1,2, \ldots$, starting from the initial conditions $u_{\tau}^{0}=u_{0}, v_{\tau}^{0}=v_{0}$, $\pi_{\tau}^{0}=\pi_{0}$, and $\alpha_{\tau}^{0}=\alpha_{0}$, cf. (12). The system is decoupled in the sense that the calculation is performed separately for $\left(u_{\tau}^{k}, v_{\tau}^{k}, \pi_{\tau}^{k}\right)$ from (16a-c) and $\alpha_{\tau}^{k}$ from (16d), as mentioned already above.

This scheme exhibits energy conservation [42], which can be seen by multiplying (16b) by the mid-point velocity $v_{\tau}^{k-1 / 2},(16 \mathrm{c})$ by the inelastic-strain rate $\left(\pi_{\tau}^{k}-\pi_{\tau}^{k-1}\right) / \tau_{k}$, and $(16 \mathrm{~d})$ by $\left(\alpha_{\tau}^{k}-\alpha_{\tau}^{k-1}\right) / \tau_{k}$. For the $\mathscr{M}$-term in the former test, we use (16a) and the binomial formula to obtain an analog of the calculus $\left\langle\mathscr{M}^{\prime} \dot{v}, v\right\rangle=\frac{\mathrm{d}}{\mathrm{d} t} \mathscr{M}(v)$ as the equality

$$
\left\langle\mathscr{M}^{\prime} \frac{v_{\tau}^{k}-v_{\tau}^{k-1}}{\tau_{k}}, v_{\tau}^{k-1 / 2}\right\rangle=\left\langle\mathscr{M}^{\prime} \frac{v_{\tau}^{k}-v_{\tau}^{k-1}}{\tau_{k}}, \frac{v_{\tau}^{k}+v_{\tau}^{k-1}}{2}\right\rangle=\frac{\mathscr{M}\left(v_{\tau}^{k}\right)-\mathscr{M}\left(v_{\tau}^{k-1}\right)}{\tau_{k}},
$$

while another binomial formula for the quadratic functional $\mathscr{E}\left(\cdot, \cdot, \alpha_{\tau}^{k-1}\right)$ gives

$$
\left\langle\mathscr{E}_{(u, \pi)}^{\prime}\left(u_{\tau}^{k-1 / 2}, \pi_{\tau}^{k-1 / 2}, \alpha_{\tau}^{k-1}\right),\left(v_{\tau}^{k-1 / 2}, \frac{\pi_{\tau}^{k}-\pi_{\tau}^{k-1}}{\tau_{k}}\right) \neq \mathscr{E}\left(u_{\tau}^{k}, \pi_{\tau}^{k}, \alpha_{\tau}^{k-1}\right)-\mathscr{E}\left(u_{\tau}^{k-1}, \pi_{\tau}^{k-1}, \alpha_{\tau}^{k-1}\right) .\right.
$$

Similarly, the latter test gives

$$
\left\langle\mathscr{E}_{\alpha}^{\prime}\left(u_{\tau}^{k}, \pi_{\tau}^{k}, \alpha_{\tau}^{k-1 / 2}\right), \frac{\alpha_{\tau}^{k}-\alpha_{\tau}^{k-1}}{\tau_{k}}\right\rangle=\mathscr{E}\left(u_{\tau}^{k}, \pi_{\tau}^{k}, \alpha_{\tau}^{k}\right)-\mathscr{E}\left(u_{\tau}^{k}, \pi_{\tau}^{k}, \alpha_{\tau}^{k-1}\right)
$$

Summing (18) with (19), we can enjoy cancellation of $\pm \mathscr{E}\left(u_{\tau}^{k}, \pi_{\tau}^{k}, \alpha_{\tau}^{k-1}\right)$. Then, summing it over $k=1,2, \ldots$, we obtain a discrete analog of the energy balance (3) as an (exact!) equality, not only as an inequality. This eliminates a spurious numerical attenuation usually exhibiting by implicit discretization schemes and is helpful during coding because accidental mistakes can thus be immediately detected.

After applying still a space discretisation, the recursive scheme (16) can be implemented on computers, leading to one linear and one linear-quadratic-programming problem at each time level. Therefore, this scheme can be solved by finite (non-iterative) algorithms, is robust (i.e. numerically stable), convergent, energy conserving. It can be interpreted as a combination of the (generalized) Newmark time discretisation with staggered (fractional-step split) for the damage flow-rule, cf. also [19] for a similar scheme. Here, for the space disretisation in the examples in the following Section 5, we used just the simplest P1 finite elements. On the other hand, a more detailed numerical analysis as far as rate of convergence or error estimates is another challenge not addressed in this article, and it is standardly considered as very difficult in such highly nonlinear problems, however. 


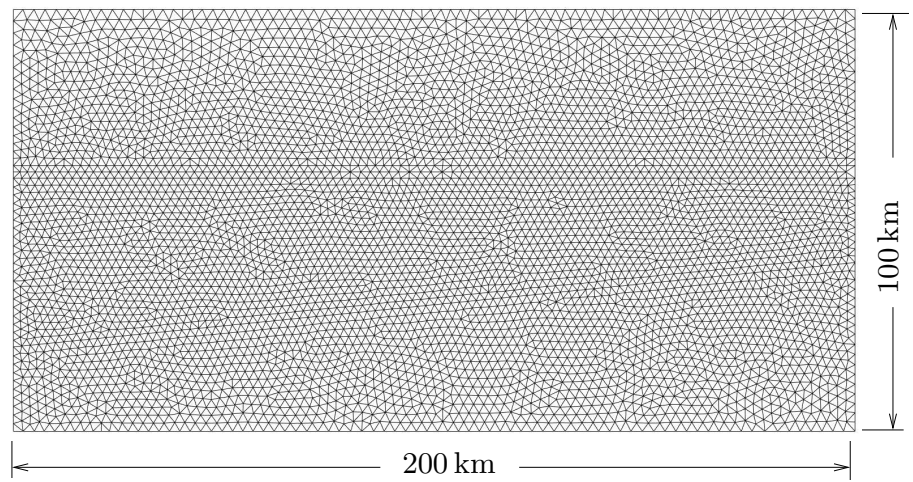

Figure 2: An example of triangular meshing of the rectangular domain used for Figure 3 below. The element size is about $2 \mathrm{~km}$.

\section{Illustrative 2D computational simulations}

We present rather academic (not entirely in real geophysical scale and only 2-dimensional) computational examples documenting efficiency and applicability range of the above presented monolithic model even in its simplified variant when $G_{M} \rightarrow \infty$ so that $\pi \rightarrow 0$. In other words, we neglect the inelastic strain, which is relevant for short-time/range events like ongoing ruptures and earthquakes around hypocentres if the (low) Maxwellian attenuation or aseismic creep are neglected. Also, for the illustrative calculations, we have neglected the viscosity, which is justified in the fluidic part (where it leads to the linear hyperbolic problem for a merely elastic fluid [40]) but not in the nonlinear solid part. Regardless, there is a belief that this shortcut does not influence substantially the presented simulations.

We consider a solid rock with the elastic bulk modulus $K_{\mathrm{ES}}=600 \mathrm{GPa}$, the shear modulus $G_{\mathrm{ES}}=250 \mathrm{GPa}$, and density $\rho_{\mathrm{S}}=5000 \mathrm{kgm}^{-3}$. Thus, in this two-dimensional situation, the speed of the $\mathrm{P}$-waves (=the sound speed) is $v_{\mathrm{PS}}=\sqrt{\left(K_{\mathrm{ES}}+G_{\mathrm{ES}}\right) / \rho_{\mathrm{S}}}=13.04 \mathrm{kms}^{-1}$, and the speed of the S-waves is $v_{\mathrm{SS}}=\sqrt{G_{\mathrm{ES}} / \rho_{\mathrm{S}}}=7.07 \mathrm{kms}^{-1}$. As for the fluids, we consider the elastic bulk modulus $K_{\mathrm{EF}}=1100 \mathrm{GPa}$, and density $\rho_{\mathrm{F}}=11000 \mathrm{kgm}^{-3}$ which provides the speed of the P-waves $v_{\mathrm{PF}}=\sqrt{K_{\mathrm{EF}} / \rho_{\mathrm{F}}}=10 \mathrm{kms}^{-1}$. The other parameters of the model which influence the damage propagation are $G_{\mathrm{c}}=2 \mathrm{Jm}^{-2}, \varepsilon=10^{-5} \mathrm{~m}, \varepsilon_{0}=1 \mathrm{~m}$.

As already emphasized, the model is truly academical. A real 3-dimensional global seismic model involving larger domains (or possibly the whole multilayered globe) should use sophisticated discretisation techniques and powerful supercomputing facilities.

Here, we present three 2-dimensional computational experiments on a rather local domain spanning "only" hundred(s) of kilometers. Therefore, instead of zero-traction boundary condition (11a), we consider boundary conditions allowing for a loading evolving in time. In particular, the load is controlled by Dirichlet boundary conditions (one can check it in Figs.3,6 and 8 below): the prescribed nonzero displacements increase at the velocity $v_{\mathrm{g}}=1 \mathrm{mms}^{-1}$. Moreover, the coefficient 3 in (6)-(8) and (10b) is to be 2 in the two-dimensional setting. Also in these three calculations, the interface between solid and fluid is considered distinctly, with the intent to provide various possibilities for reflected/refracted waves. Nevertheless, it should be emphasized that in all three calculations the same computational model is used and the differences appear only in initial/boundary conditions and geometry of the solid and the fluid phases.

The space discretisation of the 2-dimensional body made by the P1 finite elements includes an irregular but more or less uniformly sized mesh, whose smallest element size $h_{\min }=2 \mathrm{~km}$ is a hundredth of the domain dimension, cf. Figure 2. The size of the elements $h_{\min }$ is required at the interface between solid and fluid and also on pre-existing faults. The largest elements close to the top face are only a half larger. The size of the mesh (about $2 \mathrm{~km}$ ) was chosen in accordance with computational hardware (namely Intel(R) Core(TM) i5 CPU $2.3 \mathrm{GHz}$ and 8GB RAM) used to compromise the visualization of the expected geophysical events with CPU time and memory needed. Although, the shown mesh pertains to the first calculations. the second and the third ones are very similar with the same smallest element size $h_{\min }$ so that they are not shown explicitly.

The time discretisation (16) is implemented with adaptively varying time step $\tau_{k}$ shrinking when fast rupture and wave propagation start. To capture the latter phenomenon properly, the so-called Courant-Friedrich-Lewy (CFL) condition [11] has been respected, i.e. here $\tau_{k}<h_{\min } / \max \left(v_{\mathrm{PS}}, v_{\mathrm{PF}}\right)$; in fact, the CFL-condition is ultimately needed 


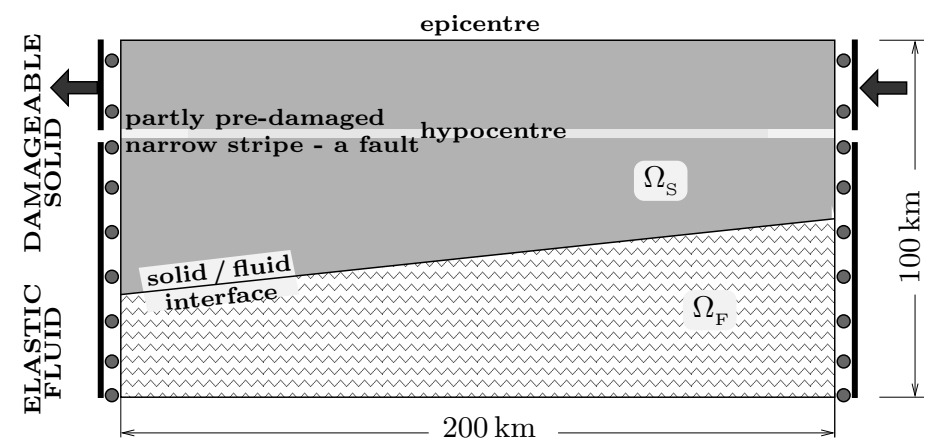

Figure 3: A computational 2-dimensional domain and boundary conditions for the experiment in Sect. 5.1. Upper trapezoidal part is the solid from Figure 1/left-part while the lower part is the fluid from Figure 1/right-part. The upper solid part is shifted by the boundary conditions to the left while the lower solid/fluid part is horizontally constrained. At time $t=0$, the fault is "compact" only at the middle part (where the hypocentre of an earthquake will be) while the rest of the fault (i.e. both sides) is (partly) damaged.

for explicit time discretisation to ensure stability and convergence but also desired for our implicit one when waves are emitted and propagating. In particular, we took $\tau_{k}=100 \mathrm{~s}$ for the initial phase of the prescribed loading and then decreased to $\tau_{k}=0.146 \mathrm{~s}$ when the rupture and wave propagation were triggered. In absolute values, the time instant $t$ of the switch to the shorter time step was chosen as follows: the first calculation $t=32 \mathrm{ks}$, the second calculation $t=49 \mathrm{ks}$, the third calculation $t=27.2 \mathrm{ks}$ according to the rupture triggering. The scale of temporal events in the experiments is then more then $2 \times 10^{5}$ in the performed academic calculations. In real structures it can be several orders higher. The prescribed horizontal shift of the upper plate at the moment of rupture initiation regarding the velocity $v_{\mathrm{g}}$ is in any case more then $27 \mathrm{~m}$ which is almost $10^{4}$ smaller then the size of the considered domain.

\subsection{Rupture on pre-existing horizontal fault}

The most typical scenario leading to tectonic earthquakes is that a fault (as a flat usually straight stripe with weakened damage threshold) is gradually stretched until the shear stress reaches the critical value to trigger the rupture. Then a fast shift occurs, within which a seismic (mainly S-) wave can be emitted. The geometry of the 2-dimensional computation region with the (a bit hypothetically) horizontally positioned fault together with boundary conditions is depicted in Figure 3; the boundary conditions imposes a prescribed displacement applied from both sides of the top solid layer.

The results of this example are depicted in Figure 4 in eight selected snapshots after rupture triggering, showing the spatial distribution of the kinetic and the stored energy in its decomposition to the shear and the spherical parts. The instants are expressed only by increments with respect to the first one $t_{1}$ due to the large time scale of the whole calculation. The actual number of numerical time steps can be computed by doing the increment by $\tau_{k}=0.146$ $\mathrm{s}$, which means e.g. 10 time steps between $t_{1}$ and $t_{2}$. The interesting moment is when the upper plate is moved sufficiently far towards left and thus the fault is stretched enough so that the rupture occurs in the middle; i.e. the earthquake starts in the hypocentre. In fact, the fracture toughness $G_{\mathrm{c}}$ is put higher close to the boundary $\Gamma$ to prevent rupture starting from the boundary where there is necessarily a stress concentration due to the fast varying boundary conditions.

At that fault rupture moment, the strain energy around is relaxed and a seismic wave is emitted and starts propagating through the solid part. One can see that this wave is not a ball-shaped partly because the seismic source (the rupturing area) is rather a surface than a point and partly (or mainly) because the seismic source by a slip of the fault generates rather an S-wave towards normal (i.e. here vertical) directions (which are slower), while the horizontallike fronts are rather P-waves (propagating faster). This is also shown in Fig. 5-left. The pertinent waves in kinetic energies around the hypocentre are shown for the snapshot corresponding to the time $t_{4}$. The P-wave is sufficiently separated from the S-wave. This is also shown in Fig. 5-left. The pertinent waves in kinetic energies around the hypocentre are shown for the snapshot corresponding to the time $t_{4}$, the detail reveals the S-wave which can also be seen for the same time instant in the shear energy in Fig. 4-middle. The P-wave is well separated from the S-wave. Here we advantageously exploit that in computer we have at disposal the strain energy split into the spherical and 

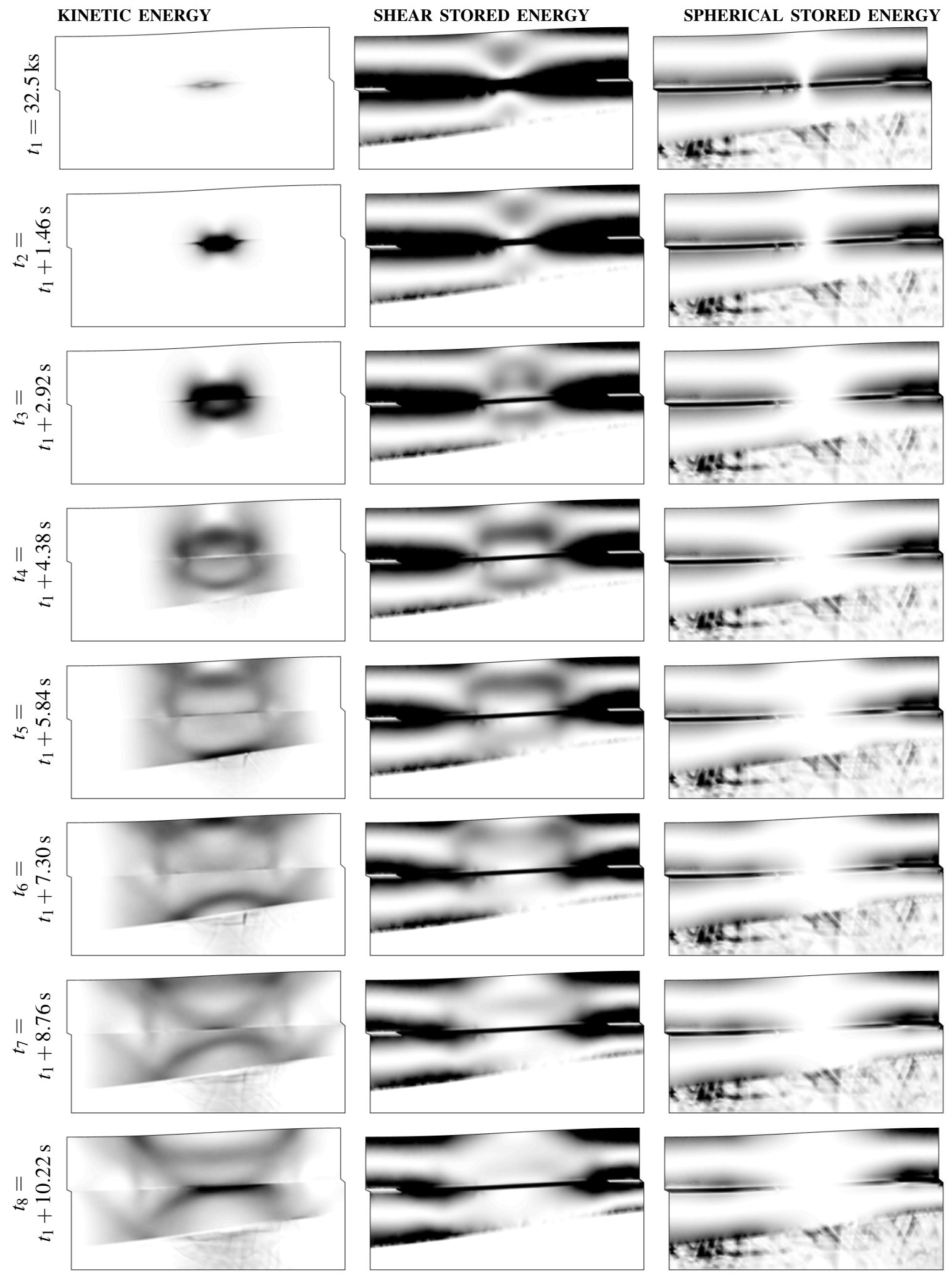

Figure 4: Simulations of a rupture of a fault in the middle part, emission of a seismic (mainly $S$-) wave in the hypocentre, its propagation and reflection on the solid/fluid interface where a weak $P$-wave can be observed in the fluid part, and eventually $S$-wave reaches also the surface (in an so-called epicentre) where the earthquake manifests. The displacement is magnified $100 \times$ to visualize the deformation. 

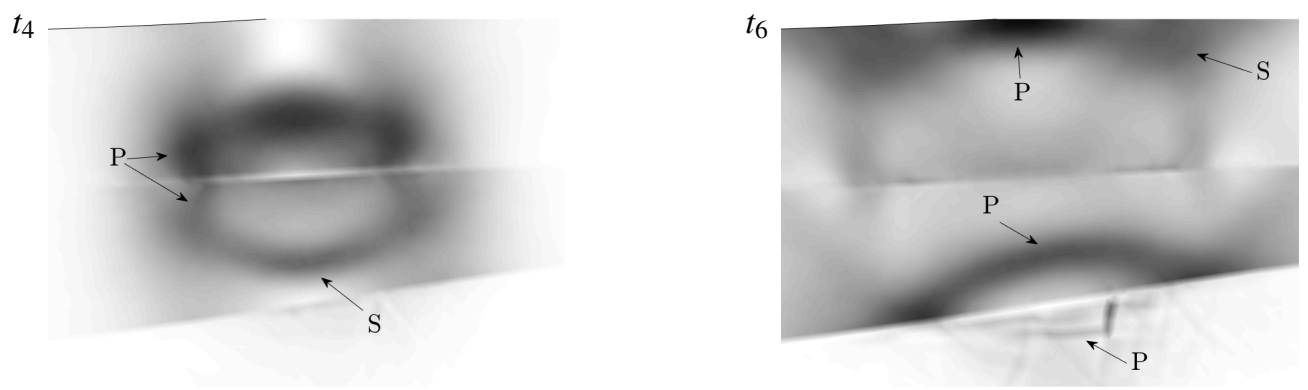

Figure 5: Various types of seismic waves recognizable in the kinetic energy drawings for the relevant parts from two selected snapshots from Fig. 4-left. The specification is possible due to the shear energy (Fig. 4-middle) where only $S$-waves can be visible, while not the $P$-waves.

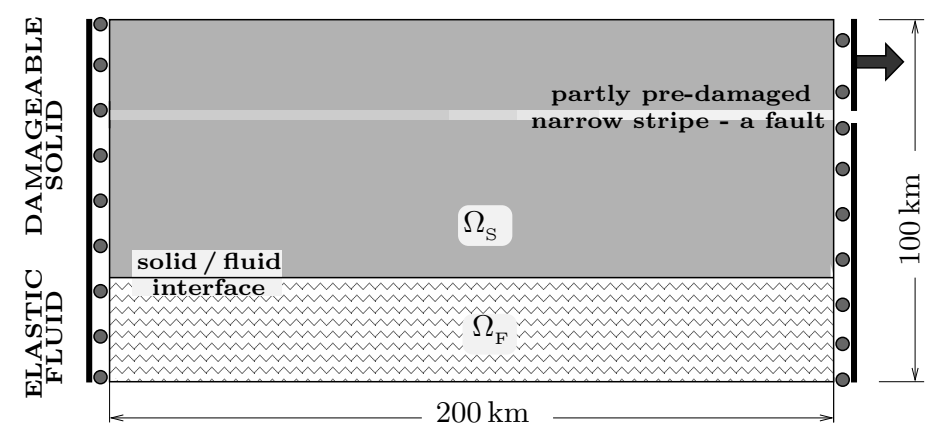

Figure 6: A computational 2-dimensional domain and boundary conditions for the experiment in Sect. 5.2. At time $t=0$, the fault is "compact" at the left part while the right part is substantially damaged.

the shear parts, from which one can distinguish these types of waves; in particular, the shear energy in Fig. 4-middle shows clearly the S-wave.

When the seismic wave reaches the Earth surface, it is reflected and partly starts propagating to the sides, i.e. the earthquake starts at the epicentre. On the other side, when reaching the solid/fluid interface below the hypocentre, the wave is mainly reflected (which documents that it is rather an S-wave) and to a small extent it generates a slight P-wave in the fluidic part. Some other reflection is seen on the fault itself, which is actually a well recognized phenomenon related to (or serving for identifying of) the width of the low-velocity damaged fault zone, cf. e.g. [4]. The detail plot in Fig. 5-right specifies the form of the reflected waves for the snapshot corresponding to the time $t_{6}$ comparing it at least with the shear energy in Fig. 4-middle at the same time instant.

\subsection{A new listric normal fault birth}

Another interesting event, although very rare from the mankind time scale, is a nucleation of damage in compact rocks, giving rise to a new damage regions, i.e. to a new fault. The geometry of the 2-dimensional computation region together with the boundary conditions imposing (unlike the previous example) the increasing displacement only at the right face of the top solid layer is depicted in Figure 6. It differs from the previous example also by the initial conditions which makes the fault largely damaged on its right-hand part. Therefore, the upper plate can quite easily slide onto the lower plate while being rather well connected with it on the left-hand part.

The results of this example are depicted in Figure 7 in seven selected snapshots of spatial distribution similar to the previous example. When the upper plate is stretched enough, it starts rupturing on an a-priori not pre-defined place. The energetics of the model dictates that the new damaging area is a (relatively) narrow plane which is positioned at about 45 degrees with respect to the existing fault. Such position, together with the slip orientation, is referred to as a normal fault, in contrast to reverse (thrust) faults, or strike or vertical faults. In fact, the new fault plane slightly curves, the dip being steeper near the surface while shallower with increased depth. This is referred to as a listric 
normal fault.

The rupture starts on the existing fault due to a slight stress concentration and then propagates towards the Earth's surface with an increasing speed, emitting a seismic wave. This wave propagates mainly through the upper plate (as an S-wave which can be observed in the plots of kinetic and shear stored energy for the time instants $t_{4}$ and $t_{5}$ ) but partly penetrated through the existing horizontal fault into the lower plate and then even towards the fluidic layer where it creates a relatively strong P-wave, cf. Figure 7-left the time instant $t_{7}$.

\subsection{A new listric reverse fault birth followed by a horizontal fault rupture}

Another interesting situation, which may occur together with a dip-slip fault as shown in the previous example, is arising of a reverse (thrust) fault. The geometry of the 2-dimensional computation region together with the boundary conditions imposing the increasing displacement at both lateral faces of the top solid layer but in opposite direction is depicted in Figure 8. It differs from the previous example also by the initial damage conditions where both ends of the fault are largely damaged. Therefore, the upper plate easily slides on the lower plate at its side parts while the central part is well connected with the lower plate and allow for stress concentration.

The results of this example are shown in Figure 9 in eight selected snapshots of spatial distribution similarly as in the previous examples. When the upper plate is substantially compressed, it starts rupturing on an a-priori not predefined place. The energetics of the model dictates that the new damaging area is a (relatively) narrow plane which is positioned in about 60 degrees with respect to the existing fault. Such position, together with the slip orientation, is referred to as a reverse (thrust) fault, here again a bit curved (listric) like in the previous normal-fault case. During this first rupture, the newly born thrust fault starts on the existing horizontal fault and then propagates towards the Earth surface with an increasing speed, emitting a seismic wave. This wave propagates mainly through the upper plate but partly penetrates through the existing horizontal fault into the lower plate and then even towards the fluidic layer where it creates a relatively strong P-wave, cf. Figure 9-left.

After a certain time (determined rather by evolving boundary conditions), it causes the horizontal fault rupture with emission of another S-waves which propagates/reflects in a similar way as in the first example in Sect. 5.1.

\section{Modifications or extensions}

The mentioned academical character of the presented, rather simple model can be suppressed when enhancing it by various ways.

\subsection{Towards easier earthquake nucleation}

It is a well recognized effect that the Griffith fracture model, which is approximated by the ansatz (8) if $\varepsilon \rightarrow 0$, is realistic as far as fracture (cracks) propagation but has difficulties with nucleation of cracks.

This effect can be seen when calculating the damage driving force $\varphi_{\alpha}^{\prime}$ used in (8), obtaining $G_{\mathrm{E}}^{\prime}(\alpha)\left|e_{\mathrm{dev}}\right|^{2}+$ $\gamma_{\mathrm{DAM}}^{\prime}(\alpha)=2(1-\alpha) G_{\mathrm{E} 0}\left|e_{\mathrm{dev}}\right|^{2}+G_{\mathrm{c}} \alpha / \varepsilon$. Counting with the shear stress $\sigma_{\mathrm{dev}}:=\operatorname{dev} \varphi_{e}^{\prime}=2 G_{\mathrm{E}}(\alpha) e_{\mathrm{dev}}$, one can see that the stress needed for triggering damage (rupture) indeed growths like $1 / \sqrt{\varepsilon}$ for $\varepsilon \rightarrow 0$, which causes the mentioned drawback of the limit Griffith-type model.

One way to get rid of this drawback is to consider, instead of $G_{\mathrm{E}}(\alpha)=\left(\varepsilon^{2} / \varepsilon_{0}^{2}+(1-\alpha)^{2}\right) G_{\mathrm{E} 1}$ used in (8), a more general convex decreasing nonlinearity $G_{\mathrm{E}}:[0,1] \rightarrow(0, \infty)$ depending on $\varepsilon$ used in the crack surface density in (8), cf. e.g. [9]. When requiring $G_{\mathrm{E}}^{\prime}(0)$ to blow up like $1 / \varepsilon$, one can still consider $\varepsilon>0$ small to imitate the narrow crack zones while keeping both nucleation and propagation under control through two mutually independent parameters $G_{\mathrm{E}}^{\prime}(0)$ and $G_{\mathrm{c}}$. Such softening may lead to localization of damage even without considering the Ambrosio-Tortorelli model, as shown by [2].

Another worthy modification which facilitates nucleation of rupture is to make $e_{\mathrm{el}}=e-\pi \mapsto \varphi(e, \pi, \alpha)$ nonconvex if $\alpha$ is close to 1 , reflecting the lost of stability of the rock when damage is sufficiently developed. The 2 -homogeneous ansatz suggested by [34] and used often in geophysical models is

$$
\varphi(e, \pi, \alpha)=\frac{\lambda_{\mathrm{E}}}{2}|\operatorname{sph} e|^{2}+G_{\mathrm{E}}(\alpha)|e-\pi|^{2}-\alpha \gamma_{\mathrm{R}}|e-\pi| \operatorname{sph} e
$$

with $\lambda_{\mathrm{E}}>0$ the first Lamé constant (fixed) and with the elastic shear modulus given by an affine relation $G_{\mathrm{E}}(\alpha)=$ $G_{0}-\alpha G_{\mathrm{R}}$ with undamaged shear modulus $G_{0}$ like in (8) and $0<G_{\mathrm{R}}<G_{0}$ sensitivity of the shear modulus to damage, 

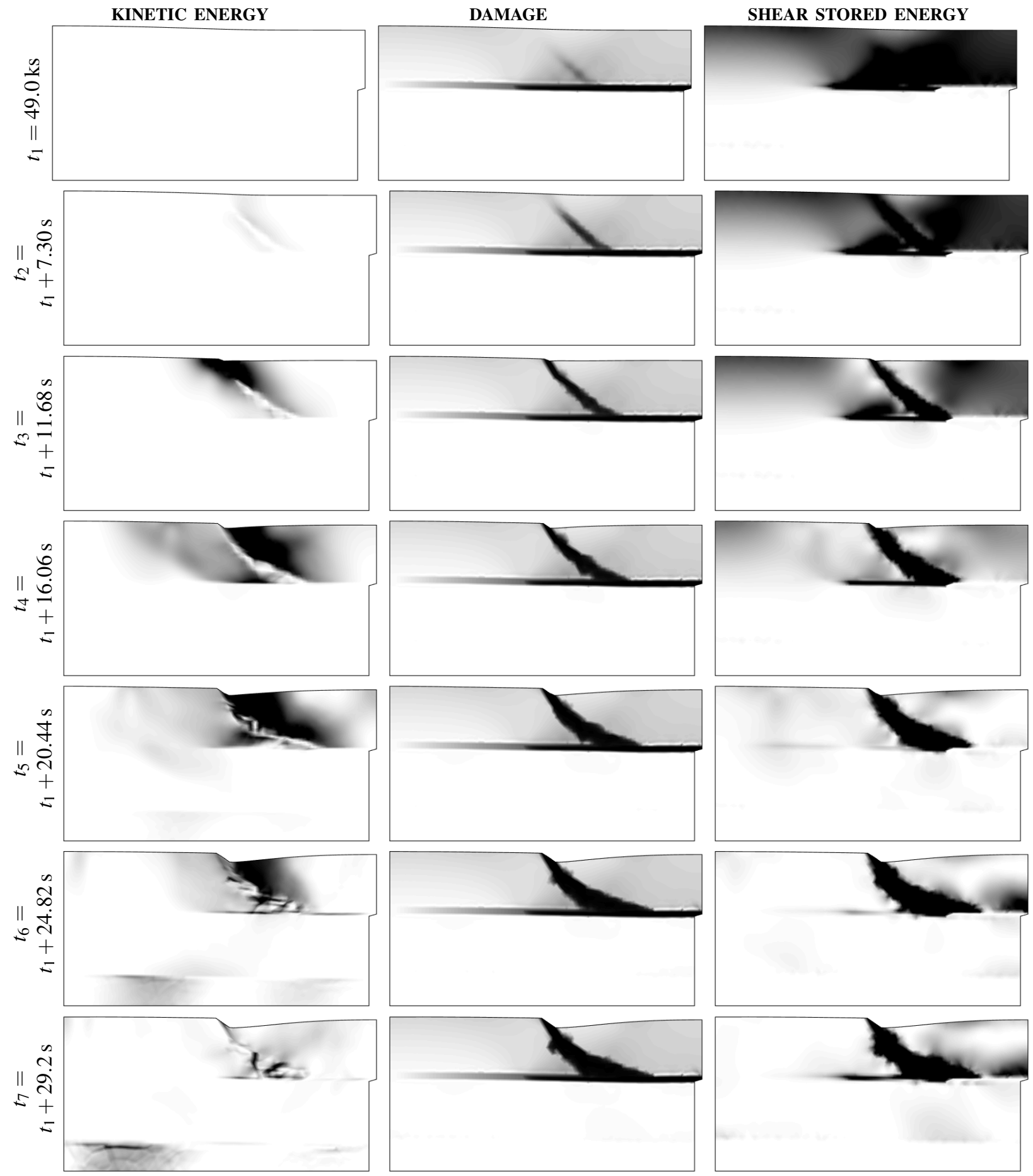

Figure 7: Simulations of a birth of a new fault in a normal position and a depression on the Earth surface, together with emission of a seismic (mainly $S$-) wave during completion of the rupture, its propagation and creation of a rather strong $P$-wave in the fluidic domain. The displacement magnified $100 \times$. 


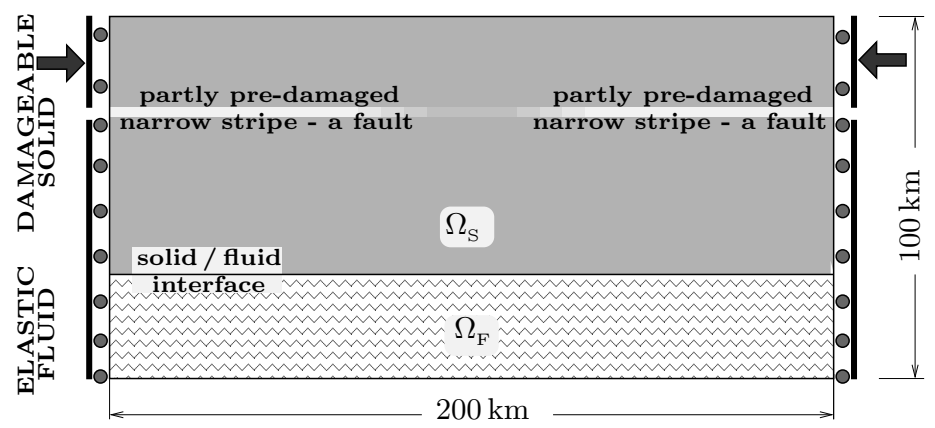

Figure 8: A computational 2-dimensional domain and boundary conditions for the experiment in Sect. 5.3. At time $t=0$, the fault is "compact" at the central part only while both the left and the right parts are substantially damaged.

while $\gamma_{R}>0$ makes it non-Hookean if the damage develops enough, cf. e.g. [16,31-33] and references therein. This leads to a nonlinear ill-posed hyperbolic problem which does not need to have any global solution in whatever sense. Its rigorous justification has been done in [39] only when one slightly modifies the ansatz (20) to reduce the fall of $\varphi$ to $-\infty$ and augment it, beside $\nabla \alpha$ and $\nabla \pi$ as in (8), also by a strain-gradient term, referring sometimes as a concept of non-simple materials. This may be related with an anomalous wave dispersion.

In both mentioned modifications that can be even combined, $\varphi$ is no longer component-wise quadratic. Therefore, in contrast to (8), the staggered scheme in Sect. 4 leads to non-quadratic minimization problems for which iterative solvers have to be used. Cf. also [41] for a brief survey of various options to improve the previous model.

\subsection{Towards other geophysical phenomena}

The set of internal variables $(\pi, \alpha)$ from Sect. 3 is a very minimal scenario to hit basic phenomena in their simplest variant. Actual modelling towards a geophysically relevant event should include more internal variables.

Putting the model into full thermodynamical context involving temperature and heat transfer would allow to include, e.g., flash heating within huge earthquakes as well as the popular Dieterich-Ruina-type rate-and-state friction model on the fault, cf. [37]. The energy-based ansatz (1) allows for thermodynamical consistency, i.e. the total-energy conservation, nonnegativity of temperature, and the Clausius-Duhem entropy inequality. In particular, the dissipation rate $\Xi=\Xi(q, \dot{q})$ from (3b) should be non-negative even locally for subsystems. In addition, $\Xi$ may depend also on temperature.

Another expansion might consider a water flow in the porous rock as in [16]. It is well known that the water content influences both the rupture process as well as the attenuation of the seismic waves. Following Biot's theory, the stored energy (6) is to be augmented as

$$
\varphi(e, \pi, \alpha, c)=\frac{3}{2} K_{\mathrm{E}}|\operatorname{sph} e|^{2}+G_{\mathrm{E}}(\alpha)|\operatorname{dev} e-\pi|^{2}+\gamma_{\mathrm{DAM}}(\alpha)+\frac{1}{2} M|\beta(\operatorname{sph} e): \mathbb{I}-(c-\phi)|^{2},
$$

where $c$ denotes the water content, and $M$ and $\beta$ are so-called Biot's modulus and Biot's coefficient, respectively, while $\phi$ denotes the porosity. Then the flow is governed by the Darcy law:

$$
\dot{c}=\operatorname{div}(m \nabla p) \quad \text { with } \quad p=M((c-\phi)-\beta(\operatorname{sph} e): \mathbb{I})
$$

where $p$ is the pore pressure and $m$ is the so-called hydraulic conductivity; note that $p=\varphi_{c}^{\prime}$ is in the position of a chemical potential and $m$ is the mobility coefficient. Also the dissipation potential (7) can be considered $c$-dependent. A staggered energy-conserving time-discretisation scheme for this complex poro-thermodynamic model has been proposed in [38]. Moreover, the porosity itself does not need to be considered fixed but may be subjected to an evolution rule similar to damage, cf. [16].

Eventually, the gravitational force $g$ in (10a) need not be considered a-priori given but rather as $g=-\rho \nabla \phi$, resulting from a gradient of the gravitational potential $\phi$ satisfying

$$
\Delta \phi=4 \pi \mathfrak{g}(\rho-\operatorname{div}(\rho u))
$$




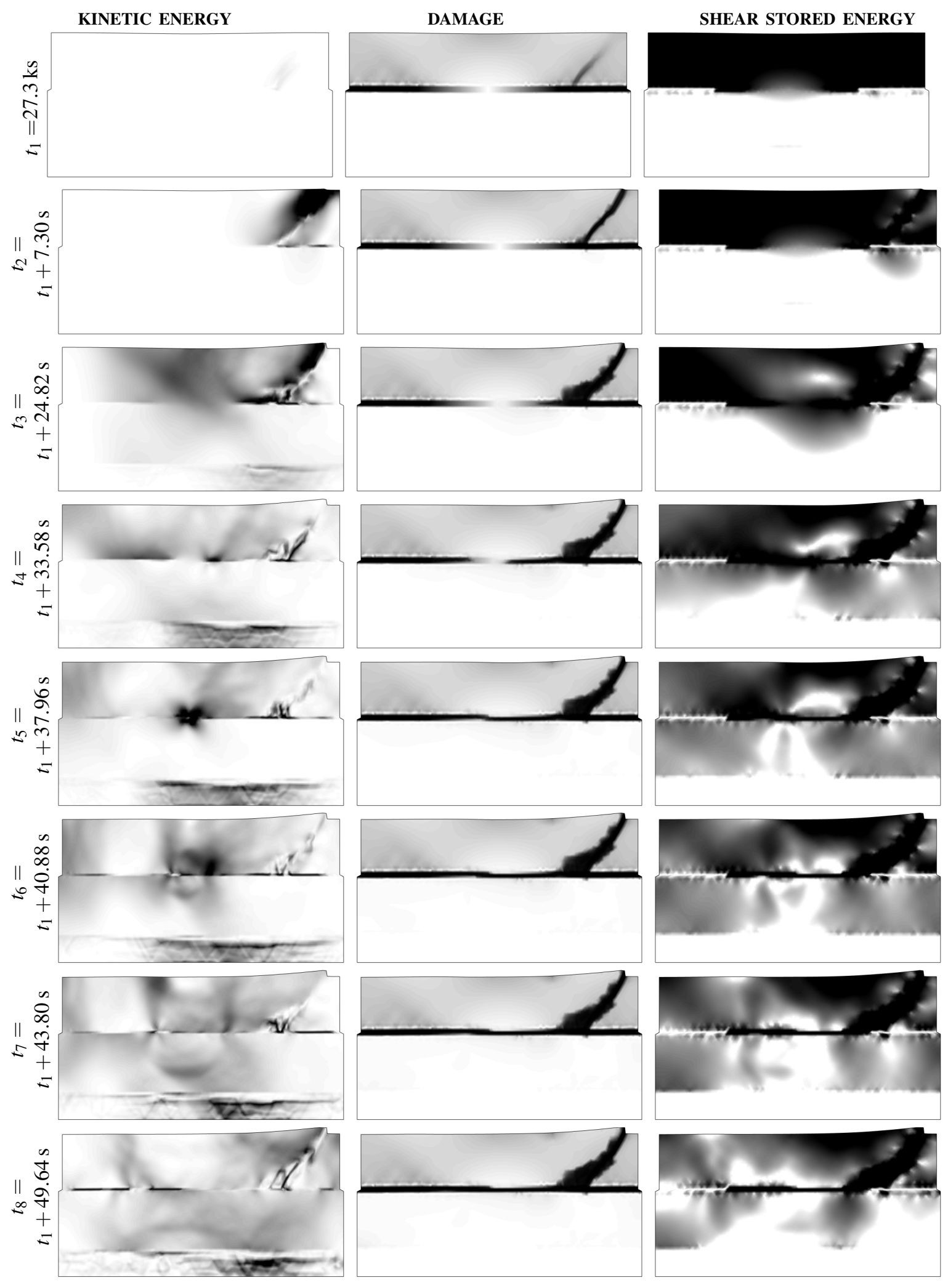

Figure 9: Simulations of a birth of a new reverse (thrust) fault in combination with a pre-existing strike-slip fault rupturing with a certain delay, together with emission of seismic (mainly $S$-) waves during completion of these two subsequenct ruptures, their propagation and creation of rather strong $P$-waves in the fluidic domain. The displacement again magnified $100 \times$ and the new mountain build during the reverse fault birth is visible. 
considered on the whole universe with the "boundary" condition $\left.\phi\right|_{|x|=\infty}=0$ and with gravitational constant $\mathfrak{g} \doteq$ $6.674 \times 10^{-11} \mathrm{~m}^{3} \mathrm{~kg}^{-1} \mathrm{~s}^{-2}$. This model of self-gravitating planet is relevant in ultra low frequency vibrations, and can be completed with considering also centrifugal and Coriolis forces. Thus the overall bulk force is

$$
g=-\rho(\nabla \phi+2 \omega \times \dot{u}+\omega \times(\omega \times(x+u)))
$$

with a given angular velocity $\omega \in \mathbb{R}^{3}$ and the vectorial cross product " $\times$ ".

\subsection{Towards bridging the scales}

Some applications (and in particular those in geophysics of solid Earth) are heavily multi-scaled as far ar both time and space concerns. Coping with this phenomenon is, numerically, very demanding and no universally applicable hints exist. Our staggered implicit scheme is well compatible with local mesh refinement which may handle spatial scales, e.g., on (and near) the fracturing surfaces (faults). Also, it is well compatible with the time-stepping refinement in (and near) the moments of ruptures and subsequent wave propagation. On the other hand, the implicit schemes ultimately require solving large systems of algebraic equations, which brings computational restrictions. In particular, when waves are propagating through large distances, one needs a fine mesh (or high-order finite elements) everywhere. For this reason, rather explicit time-discretisations are used, which ultimately, however, require the mentioned CFL condition even during periods when only slow-loading processes are on effect without any rupturing and wave emission and which makes usage of such methods limited. Here, one may think about an adaptive combination of the implicit discretisation using rather large time-steps and admitting local mesh refinement with an explicit disretisation using short time steps but only during relatively short events (typically minutes during earthquakes versus years or thousands of years in between them). If the 2nd-order system is implemented in a staggered way as a 1st-order system, varying of time step is easy. A rigorous combination of explicit methods with implicit discretisation of damage and Maxwellian viscoelastic rheology or poroelasticity model is not trivial and has recently be devised in [43]. The spatial scales would require still several meshes: a fine for resolving waves by explicit discretisation (possibly with special so-called spectral finite elements $[21,25,26])$ while an only locally refined mesh for solving systems of equations arising from the gradient-damage model.

\subsection{Towards very long time scales}

Even solid geophysical materials (lithospheric rocks in the Earth mantle) become rather fluidic on very long time scales (millions of years). Displacements and plastic deformation can then be large even if the elastic strain is small. A compromising model still based on small strains then involves Korteweg-like stress contribution arising from the transport of internal variables, and it has been formulated in [31] and later analysed in [39]. The full thermodynamically consistent model should be formulated at large strains, cf. [44], but its numerical implementation is very cumbersome.

\section{Conclusion}

A coupled model for wave source due to fracture in elastic solid (approximated by a phase-field approach) and propagation of these waves in the layered solid and fluidic continua has been presented and tested computationally on 2-dimensional examples. The interpretation as seismic sources by tectonic earthquakes generating seismic waves and for their propagation was suggested. The ability of this model to capture basic phenomena occurring during earthquakes on pre-existing faults and during the creation of new faults has been demonstrated even when using rather basic damage-type models for the rupture.

Other, more "local" and engineering, usages might be for various destructive processes in rocks or concrete constructions adjacent to water or oil reservoirs, or in hydraulic fracture merged with natural faults (like e.g. in [24] in quasistatic variant).

The algorithmically efficient staggered time discretisation scheme has been used. This was combined with the simplest P1 finite elements for the space discretisation. Yet, it is well known that this discretisation is not optimal for elasticity and more sophisticated spectral elements [21-23,25,26] or discontinous P2-elements [45] are implemented in this context. 
Eventually, various expansions of this basic model have been outlined. In fact, Sections 2-4 already introduce another internal variable (the inelastic strain) that should be considered for long-time processes as creep or as healing during long lasting periods in between earthquakes, although it was not implemented in the presented computational simulations in Sect. 5.

\section{REFERENCES}

[1] L. Ambrosio and V. M. Tortorelli. Approximation of free discontinuity problems. Boll. Unione Mat. Italiana, 6-B:105-123, 1992.

[2] Z.P. Bažant and M. Jirásek. Softening-induced dynamic localization instability: seismic damage in frames. $J$. Engr. Mech., 122:1149-1158, 1996.

[3] A. Bedford. Hamilton's Principle in Continuum Mechanics. Pitman, Boston, 1985.

[4] Y. Ben-Zion. Properties of seismic fault zone waves and their utility for imaging low-velocity structures. $J$. Geophys. Res., 103:12,567-12,585, 1998.

[5] Y. Ben-Zion. Dynamic ruptures in recent models of earthquake faults. J. Mech. Phys. Solids, 49:2209-2244, 2001.

[6] Y. Ben-Zion and J.-P. Ampuero. Seismic radiation from regions sustaining material damage. Geophys. J. Int., 178:1351-1356, 2009.

[7] D. V. Boger. A highly elastic constant-viscosity fluid. J. Non-Newtonian Fluid Mechanics, 3:87-91, 1977.

[8] B. Bourdin, C.J. Larsen, and C.L. Richardson. A time-discrete model for dynamic fracture based on crack regularization. Int. J. of Fracture, 10:133-143, 2011.

[9] B. Bourdin, J.-J. Marigo, C. Maurini, and P. Sicsic. Morphogenesis and propagation of complex cracks induced by thermal shocks. Phys. Rev. Lett., 112:014301, 2014.

[10] E. Choi, E. Tan, L.L. Lavier, and V.M. Calo. DynEarthSol2D: An efficient unstructured finite element method to study long-term tectonic deformation. J. Geophys. Res. Solid Earth, 118:2429-2444, 2013.

[11] R. Courant, K. Friedrichs, and H. Lewy. Über die partiellen Differenzengleichungen der mathematischen Physik. Math. Annalen, 100:32-74, 1928.

[12] M. Focardi. On the variational approximation of free-discontinuity problems in the vectorial case. Math. Models Methods Appl. Sci., 11:663-684, 2001.

[13] A. Green and P. Naghdi. A general theory of an elastic-plastic continuum. Arch. Rational Mech. Anal., 18:251$281,1965$.

[14] A.A. Griffith. The phenomena of rupture and flow in solids. Phil. Trans. Royal Soc. London, A, 221:163-198, 1921.

[15] B. Halphen and Q.S. Nguyen. Sur les matériaux standards généralisés. J. Mécanique, 14:39-63, 1975.

[16] Y. Hamiel, V. Lyakhovsky, and A. Agnon. Coupled evolution of damage and porosity in poroelastic media: theory and applications to deformation of porous rocks. Geophys. J. Int., 156:701-713, 2004.

[17] W.R. Hamilton. On a general method in dynamics, part II. Phil. Trans. Royal Soc., pages 247-308, 1834.

[18] R. A. Harris et al. The SCEC/USGS dynamic earthquake rupture code verification exercise. Seismological Res. Lett., 80:119-126, 2009.

[19] M. Hofacker and C. Miehe. Continuum phase field modeling of dynamic fracture: variational principles and staggered FE implementation. Intl. J. Fracture, 178:113-129, 2012. 
[20] Y. Huang, J.-P. Ampuero, and D. V. Helmberger. Earthquake ruptures modulated by waves in damaged fault zones. J. of Geophysical Research: Solid Earth, B9:3133-3154, 2014.

[21] Y. Kaneko, N. Lapusta, and J.-P. Ampuero. Spectral element modeling of spontaneous earthquake rupture on rate and state faults: Effect of velocity-strengthening friction at shallow depths. J. Geophysical Res., 113:B09317, 2008.

[22] M. Käser and M. Dumbser. An arbitrary high-order discontinuous Galerkin method for elastic waves on unstructured meshes I: The two-dimensional isotropic case with external source terms. Geophys. J. Int., 166:855-877, 2006.

[23] M. Käser, M. Dumbser, J. de la Puente, and H. Igel. An arbitrary high-order discontinuous Galerkin method for elastic waves on unstructured meshes III: Viscoelastic attenuation. Geophys. J. Int., 168:224-242, 2007.

[24] A.R. Khoei, M. Vahab, and M. Hirmand. Modeling the interaction between fluid-driven fracture and natural fault using an enriched-FEM technique. Intl. J. Fracture, 197:1-24, 2016.

[25] D. Komatitsch and J. Tromp. Spectral-element simulations of global seismic wave propagation - I. validation. Geophys. J. Int., 149:390-412, 2002.

[26] D. Komatitsch and J. Tromp. Spectral-element simulations of global seismic wave propagation - II. threedimensional models, oceans, rotation and self-gravitation. Geophys. J. Int., 150:303-318, 2002.

[27] M. Kružík and T. Roubíček. Mathematical Methods in Continuum Mechanics of Solids. Springer, Switzerland, 2019.

[28] C.J. Larsen, C. Ortner, and E. Süli. Existence of solution to a regularized model of dynamic fracture. Math. Models Meth. Appl. Sci., 20:1021-1048, 2010.

[29] T. Lay and T. C. Wallace. Modern global seismology. Acad. Press, San Diego, 1995.

[30] V. Lyakhovsky and Y. Ben-Zion. A Continuum Damage-Breakage Faulting Model and Solid-Granular Transitions. Pure Appl. Geophysics, 171:3099-3123, 2014.

[31] V. Lyakhovsky and Y. Ben-Zion. Damage-breakage rheology model and solid-granular transition near brittle instability. J. Mech. Phys. Solids, 64:184-197, 2014.

[32] V. Lyakhovsky, Y. Hamiel, J.-P. Ampuero, and Y. Ben-Zion. Non-linear damage rheology and wave resonance in rocks. Geophys. J. Int., 178:910-920, 2009.

[33] V. Lyakhovsky, Y. Hamiel, and Y. Ben-Zion. A non-local visco-elastic damage model and dynamic fracturing. J. Mech. Phys. Solids, 59:1752-1776, 2011.

[34] V. Lyakhovsky and V.P. Myasnikov. On the behavior of elastic cracked solid. Phys. Solid Earth, 10:71-75, 1984.

[35] A. Mielke and T. Roubíček. Rate-Independent Systems - Theory and Application. Springer, New York, 2015.

[36] C. Pelties, J. de la Puente, J.-P. Ampuero, G. B. Brietzke, and M. Käser. Three-dimensional dynamic rupture simulation with a high-order discontinuous galerkin method on unstructured tetrahedral meshes. J. Geophys. Res., 117:B02309, 2012.

[37] T. Roubíček. A note about the rate-and-state-dependent friction model in a thermodynamical framework of the Biot-type equation. Geophys. J. Intl., 199:286-295, 2014.

[38] T. Roubíček. An energy-conserving time-discretisation scheme for poroelastic media with phase-field fracture emitting waves and heat. Disc. Cont. Dynam. Syst. S, 10:867-893, 2017.

[39] T. Roubíček. Geophysical models of heat and fluid flow in damageable poro-elastic continua. Cont. Mech. Thermodyn., 29:625-646, 2017. 
[40] T. Roubíček. Seismic waves and earthquakes in a global monolithic model. Cont. Mech. Thermodynam., 30:709-729, 2018.

[41] T. Roubíček. Models of dynamic damage and phase-field fracture, and their various time discretisations. In J.-F. Rodrigues and M. Hinttermüller, editors, Topics in Applied Analysis and Optimisation, CIM Series in Math. Sci. Springer, in print. Preprint arXiv 1906.04110.

[42] T. Roubíček and C. G. Panagiotopoulos. Energy-conserving time-discretisation of abstract dynamical problems with applications in continuum mechanics of solids. Numer. Funct. Anal. Optim., 38:1143-1172, 2017.

[43] T. Roubíček, C.G. Panagiotopoulos, and C. Tsogka. Explicit time-discretisation of elastodynamics with some inelastic processes at small strains . 2019. Preprint arXiv no.1903.11654.

[44] T. Roubíček and U. Stefanelli. Thermodynamics of elastoplastic porous rocks at large strains towards earthquake modeling. SIAM J. Appl. Math., 78:2597-2625, 2018.

[45] J. Tago, V. M. Cruz-Atienza, J. Virieux, V. Etienne, and F. J. Snchez-Sesma. A 3D hp-adaptive discontinuous Galerkin method for modeling earthquake dynamics. J. Geophys. Res., 117:B09312, 2012.

[46] N. Tosi, O. Čadek, and Z. Martinec. Subducted slabs and lateral viscosity variations: effects on the longwavelength geoid. Geophys. J. Int., 179:813-826, 2009. 